\title{
Double phase problems with variable growth and convection for the Baouendi-Grushin operator
}

\author{
Anouar Bahrouni(i), Vicenţiu D. Rădulescu(D and Patrick Winkert(i)
}

\begin{abstract}
In this paper we study a class of quasilinear elliptic equations with double phase energy and reaction term depending on the gradient. The main feature is that the associated functional is driven by the Baouendi-Grushin operator with variable coefficient. This partial differential equation is of mixed type and possesses both elliptic and hyperbolic regions. We first establish some new qualitative properties of a differential operator introduced recently by Bahrouni et al. (Nonlinearity 32(7):2481-2495, 2019). Next, under quite general assumptions on the convection term, we prove the existence of stationary waves by applying the theory of pseudomonotone operators. The analysis carried out in this paper is motivated by patterns arising in the theory of transonic flows.
\end{abstract}

Mathematics Subject Classification. 35J70, 35P30, 76H05.

Keywords. Baouendi-Grushin operator, Double phase problem, Convection term, Pseudomonotone operator.

\section{Introduction}

Let $\Omega \subset \mathbb{R}^{N}, N>1$, be a bounded domain with smooth boundary $\partial \Omega$ and let $n, m$ be nonnegative integers such that $N=n+m$. This means that $\mathbb{R}^{N}=\mathbb{R}^{n} \times \mathbb{R}^{m}$ and so $z \in \Omega$ can be written as $z=(x, y)$ with $x \in \mathbb{R}^{n}$ and $y \in \mathbb{R}^{m}$.

We consider the following double phase problem with convection term

$$
\begin{aligned}
-\Delta_{G(x, y)} u+A(x, y)\left(|u|^{G(x, y)-1}+|u|^{G(x, y)-3}\right) u & =f((x, y), u, \nabla u) & & \text { in } \Omega, \\
u & =0 & & \text { on } \partial \Omega,
\end{aligned}
$$

with

$$
A(x, y)=\left|\nabla_{x} G(x, y)\right|+|x|^{\gamma}\left|\nabla_{y} G(x, y)\right| \quad \text { for all }(x, y) \in \Omega .
$$

Here, $G: \bar{\Omega} \rightarrow(1, \infty)$ is supposed to be a continuous function and $\Delta_{G(x, y)}$ stands for the BaouendiGrushin operator with variable coefficient, which is defined by

$$
\begin{aligned}
\Delta_{G(x, y)} u & =\operatorname{div}\left(\nabla_{G(x, y)} u\right) \\
& =\sum_{i=1}^{n}\left(\left|\nabla_{x}\right|^{G(x, y)-2} u_{x_{i}}\right)_{x_{i}}+|x|^{\gamma} \sum_{i=1}^{m}\left(\left|\nabla_{y}\right|^{G(x, y)-2} u_{y_{i}}\right)_{y_{i}},
\end{aligned}
$$

where

$$
\nabla_{G(x, y)} u=\mathcal{A}(x)\left[\begin{array}{cc}
\left|\nabla_{x}\right|^{G(x, y)-2} & \nabla_{x} u \\
|x|^{\gamma}\left|\nabla_{y}\right|^{G(x, y)-2} & \nabla_{y} u
\end{array}\right]
$$


and

$$
\mathcal{A}(x)=\left[\begin{array}{cc}
I_{n} & 0_{n, m} \\
0_{m, n} & |x|^{\gamma} I_{m}
\end{array}\right] \in \mathcal{M}_{N \times N}(\mathbb{R}),
$$

where $I_{n}$ is the identity matrix of size $n \times n, O_{n, m}$ is the zero matrix of size $n \times m$, and $\mathcal{M}_{N \times N}$ stands for the class of $N \times N$-matrices with real-valued entries. From the representation above it is clear that $\Delta_{G(x, y)}$ is degenerate along the $m$-dimensional subspace $M:=\{0\} \times \mathbb{R}^{m}$ of $\mathbb{R}^{N}$.

The differential operator $\Delta_{G(x, y)}$ generalizes the degenerate operator

$$
\frac{\partial^{2}}{\partial x^{2}}+x^{2 r} \frac{\partial^{2}}{\partial y^{2}} \quad(r \in \mathbb{N})
$$

introduced by Baouendi [7] and Grushin [17]. The Baouendi-Grushin operator can be viewed as the Tricomi operator for transonic flow restricted to subsonic regions. On the other hand, a second-order differential operator $T$ in divergence form on the plane can be written as an operator whose principal part is a Baouendi-Grushin-type operator, provided that the principal part of $T$ is nonnegative and its quadratic form does not vanish at any point, see Franchi and Tesi [15].

In the right-hand side of problem (1.1) we have a nonlinearity $f: \Omega \times \mathbb{R} \times \mathbb{R}^{N} \rightarrow \mathbb{R}$ which is a Carathéodory function, that is, $f(\cdot, s, \xi)$ is measurable for all $(s, \xi) \in \mathbb{R} \times \mathbb{R}^{N}$ and $f((x, y), \cdot, \cdot)$ is continuous for a.a. $(x, y) \in \Omega$.

Problem (1.1) is strictly connected with the analysis of nonlinear patterns and stationary waves for transonic flow models. We refer to the pioneering work of Morawetz [20-22] on the theory of transonic fluid flow-referring to partial differential equations that possess both elliptic and hyperbolic regionsand this remains the most fundamental mathematical work on this subject. The flow is supersonic in the elliptic region, while a shock wave is created at the boundary between the elliptic and hyperbolic regions. In the 1950s, Morawetz used functional-analytic methods to study boundary value problems for such transonic problems.

The variable coefficient $G(x, y)$ describes the geometry of a composite realized by using two materials with corresponding behaviour described by $\left|\nabla_{x} u\right|^{G(x, y)}$ and $\left|\nabla_{y} u\right|^{G(x, y)}$. Then in the region $\{z \in \Omega: x \neq$ $0\}$ the material described by the second integrand is present. In the opposite case, the material described by the first integrand is the only one that creates the composite.

The main goal of our paper is to prove the existence of at least one weak solution of problem (1.1) under very general conditions on the nonlinearity $f: \Omega \times \mathbb{R} \times \mathbb{R}^{N} \rightarrow \mathbb{R}$. The novelty of our paper is the fact that we combine a double phase operator driven by the Baouendi-Grushin operator with variable growth and a right-hand side which depends on the gradient of the solution. Such function is called convection term.

It is well known that the Caffarelli-Kohn-Nirenberg inequality is a powerful inequality and it is needed in several ways in the study of partial differential equations. We refer to the works of Adimurthi et al. [2], Baroni et al. [8], Colasuonno and Pucci [12], Colombo and Mingione [13] for relevant applications of the Caffarelli-Kohn-Nirenberg inequality. For recent contributions to the study of double-phase problems we refer to Beck and Mingione [9], Papageorgiou et al. [24,25], and Zhang and Rădulescu [31].

The following Caffarelli-Kohn-Nirenberg inequality [10] establishes that for given $p \in(1, N)$ and real numbers $a, b$ and $q$ such that

$$
-\infty<a<\frac{N-p}{p}, \quad a \leq b \leq a+1, \quad q=\frac{N p}{N-p(1+a-b)},
$$

there exists a positive constant $C_{a, b}$ such that for all $u \in C_{c}^{1}(\Omega)$

$$
\left(\int_{\Omega}|x|^{-b q}|u|^{q} \mathrm{~d} x\right)^{p / q} \leq C_{a, b} \int_{\Omega}|x|^{-a p}|\nabla u|^{p} \mathrm{~d} x .
$$


This inequality was extensively studied, see for example Abdellaoui and Peral [1], Adimurthi et al. [2], Bahrouni et al. [4,5], Catrina and Wang [11], and the references therein. In particular, Bahrouni et al. [5] proved a new version of a Caffarelli-Kohn-Nirenberg inequality with variable exponent for the Baouendi-Grushin operator $\Delta_{G}$. More precisely, the following weighted inequality has been proved.

Theorem 1.1. Assume that $G$ is a function of class $C^{1}$ and that $G(x, y) \in(2, N)$ for all $(x, y) \in \Omega$. Then there exists a positive constant $\beta$ such that for all $u \in C_{c}^{1}(\Omega)$

$$
\begin{aligned}
& \int_{\Omega}\left(1+|x|^{\gamma}\right)|u|^{G(x, y)} \mathrm{d} x \mathrm{~d} y \\
& \quad \leq \beta \int_{\Omega}\left(\left|\nabla_{x} u\right|^{G(x, y)}+|x|^{\gamma}\left|\nabla_{y} u\right|^{G(x, y)}\right) \mathrm{d} x \mathrm{~d} y \\
& \quad+\beta \int_{\Omega}|u|^{G(x, y)-1}\left(1+u^{2}\right)\left(\left|\nabla_{x} G(x, y)\right|+|x|^{\gamma}\left|\nabla_{y} G(x, y)\right|\right) \mathrm{d} x \mathrm{~d} y .
\end{aligned}
$$

The paper is organized as follows. In Sect. 2 we present the basic properties of variable Lebesgue and Sobolev spaces and state the main tools which will be used later; see Rădulescu and Repovš [29] for more details. New properties concerning the Baouendi-Grushin operator will be discussed in Sect. 3, and in the last section we state and prove our main result concerning the existence of a weak solution to problem (1.1).

\section{Terminology and the abstract setting}

In this section we recall some basic definitions and properties of the needed function spaces. We refer to the works of Bahrouni and Repovš [6], Hájek et al. [18], Musielak [23], Rădulescu [27,28], Rădulescu and Repovš [29] and the references therein. Consider the set

$$
C_{+}(\bar{\Omega})=\{p \in C(\bar{\Omega}) \mid p(x)>1 \text { for all } x \in \bar{\Omega}\}
$$

and define for any $p \in C_{+}(\bar{\Omega})$

$$
p^{+}:=\sup _{x \in \bar{\Omega}} p(x) \quad \text { and } \quad p^{-}:=\inf _{x \in \bar{\Omega}} p(x) .
$$

Then $1<p^{-} \leq p^{+}<\infty$ for each $p \in C_{+}(\bar{\Omega})$. The variable exponent Lebesgue space $L^{p(\cdot)}(\Omega)$ is defined by

$$
L^{p(\cdot)}(\Omega)=\left\{u: \Omega \rightarrow \mathbb{R} \mid u \text { is measurable and } \int_{\Omega}|u(x)|^{p(x)} \mathrm{d} x<\infty\right\}
$$

equipped with the Luxemburg norm

$$
\|u\|_{p(\cdot)}=\inf \left\{\mu>\left.0\left|\int_{\Omega}\right| \frac{u(x)}{\mu}\right|^{p(x)} \mathrm{d} x \leq 1\right\} .
$$

It is known that $L^{p(\cdot)}(\Omega)$ is a reflexive Banach space. Moreover, continuous functions with compact support are dense in $L^{p(\cdot)}(\Omega)$.

Denote by $q(\cdot)$ the conjugate of $p(\cdot)$, that is, $1 / p(x)+1 / q(x)=1$ for all $x \in \bar{\Omega}$. If $u \in L^{p(\cdot)}(\Omega)$ and $v \in L^{q(\cdot)}(\Omega)$, then we have the following Hölder-type inequality

$$
\left|\int_{\Omega} u v \mathrm{~d} x\right| \leq\left(\frac{1}{p^{-}}+\frac{1}{q^{-}}\right)\|u\|_{p(\cdot)}\|v\|_{q(\cdot)} .
$$


More generally, if $p_{j} \in C_{+}(\bar{\Omega})$ for $j=1,2,3$ and

$$
\frac{1}{p_{1}(x)}+\frac{1}{p_{2}(x)}+\frac{1}{p_{3}(x)}=1 \quad \text { for all } x \in \bar{\Omega}
$$

then we obtain for all $u \in L^{p_{1}(\cdot)}(\Omega), v \in L^{p_{2}(\cdot)}(\Omega)$ and $w \in L^{p_{3}(\cdot)}(\Omega)$ that

$$
\left|\int_{\Omega} u v w \mathrm{~d} x\right| \leq\left(\frac{1}{p_{1}^{-}}+\frac{1}{p_{2}^{-}}+\frac{1}{p_{3}^{-}}\right)\|u\|_{p_{1}(\cdot)}\|v\|_{p_{2}(\cdot)}\|w\|_{p_{3}(\cdot)} .
$$

Moreover, for $p_{1} \leq p_{2}$ in $\Omega$, then there exists the continuous embedding $L^{p_{2}(\cdot)}(\Omega) \hookrightarrow L^{p_{1}(\cdot)}(\Omega)$.

The following two propositions will be useful in the sequel.

Proposition 2.1. Let

$$
\rho_{1}(u)=\int_{\Omega}|u|^{p(x)} \mathrm{d} x \quad \text { for all } u \in L^{p(\cdot)}(\Omega) .
$$

Then the following holds:

(i) $\|u\|_{p(\cdot)}<1$ (resp., =1;>1) if and only if $\rho_{1}(u)<1$ (resp., =1; $>1$ );

(ii) $\|u\|_{p(\cdot)}>1$ implies $\|u\|_{p(\cdot)}^{p^{-}} \leq \rho_{1}(u) \leq\|u\|_{p(\cdot)}^{p^{+}}$;

(iii) $\|u\|_{p(\cdot)}<1$ implies $\|u\|_{p(\cdot)}^{p^{+}} \leq \rho_{1}(u) \leq\|u\|_{p(\cdot)}^{p^{-}}$.

Proposition 2.2. Let

$$
\rho_{1}(u)=\int_{\Omega}|u|^{p(x)} \mathrm{d} x \quad \text { for all } u \in L^{p(\cdot)}(\Omega) .
$$

If $u, u_{n} \in L^{p(\cdot)}(\Omega)$ and $n \in \mathbb{N}$, then the following statements are equivalent:

(i) $\lim _{n \rightarrow+\infty}\left\|u_{n}-u\right\|_{p(\cdot)}=0$;

(ii) $\lim _{n \rightarrow+\infty} \rho_{1}\left(u_{n}-u\right)=0$;

(iii) $u_{n}(x) \rightarrow u(x)$ in $\Omega$ and $\lim _{n \rightarrow+\infty} \rho_{1}\left(u_{n}\right)=\rho_{1}(u)$.

By $W^{1, p(\cdot)}(\Omega)$ we denote the variable exponent Sobolev space

$$
W^{1, p(\cdot)}(\Omega)=\left\{u \in L^{p(\cdot)}(\Omega)|| \nabla u \mid \in L^{p(\cdot)}(\Omega)\right\}
$$

equipped with the norm

$$
\|u\|_{1, p(\cdot)}=\|\nabla u\|_{p(\cdot)}+\|u\|_{p(\cdot)}
$$

Then $W^{1, p(\cdot)}(\Omega)$ is a reflexive and separable Banach space.

Our main existence result will be based on the following surjectivity result, see Gasinski and Papageorgiou [16]. First, we give the definition of pseudomonotonicity.

Definition 2.3. Let $X$ be a reflexive Banach space, $X^{*}$ its dual space and denote by $\langle\cdot, \cdot\rangle$ its duality pairing. Let $A: X \rightarrow X^{*}$, then $A$ is called pseudomonotone if $u_{n} \stackrel{\mathrm{w}}{\rightarrow} u$ in $X$ and $\limsup _{n \rightarrow \infty}\left\langle A\left(u_{n}\right), u_{n}-u\right\rangle \leq 0$ imply $A u_{n} \stackrel{\mathrm{w}}{\rightarrow} u$ and $\left\langle A u_{n}, u_{n}\right\rangle \rightarrow\langle A u, u\rangle$.

Theorem 2.4. Let $X$ be a real, reflexive Banach space, and let $A: X \rightarrow X^{*}$ be a pseudomonotone, bounded, and coercive operator, and $b \in X^{*}$. Then the problem $A u=b$ has at least one solution. 


\section{Properties of the double phase operator and the corresponding function space}

In this section we recall and prove new results concerning the Baouendi-Grushin operator introduced in Sect. 1.

Based on Theorem 1.1, we denote by $\mathcal{W}$ the closure of $C_{c}^{1}(\Omega)$ with respect to the norm

$$
\begin{aligned}
\|u\|= & \left\|\nabla_{x} u\right\|_{G(\cdot, \cdot)}+\left\||x|^{\frac{\gamma}{G(\cdot, \cdot)}} \nabla_{y} u\right\|_{G(\cdot, \cdot)} \\
& +\left\|u\left(\left|\nabla_{x} G(x, y)\right|+|x|^{\gamma}\left|\nabla_{y} G(x, y)\right|\right)^{\frac{1}{G(x, y)+1}}\right\|_{G(\cdot, \cdot)+1} \\
& +\left\|u\left(\left|\nabla_{x} G(x, y)\right|+|x|^{\gamma}\left|\nabla_{y} G(x, y)\right|\right)^{\frac{1}{G(x, y)-1}}\right\|_{G(\cdot, \cdot)-1} .
\end{aligned}
$$

Note that the norm $\|\cdot\|$ on $\mathcal{W}$ is equivalent to

$$
\begin{aligned}
& \|u\|_{\mathcal{W}} \\
& =\inf \left\{\mu \geq 0 \mid \rho\left(\frac{u}{\mu}\right) \leq 1\right\} \\
& =\inf \left\{\mu \geq 0 \mid \int_{\Omega} \frac{1}{G(x, y)}\left[\left|\nabla_{x}\left(\frac{u}{\mu}\right)\right|^{G(x, y)}+|x|^{\gamma}\left|\nabla_{y}\left(\frac{u}{\mu}\right)\right|^{G(x, y)}\right] \mathrm{d} x \mathrm{~d} y\right. \\
& \left.\quad+\int_{\Omega} A(x, y)\left[\frac{\left|\frac{u}{\mu}\right|^{G(x, y)+1}}{G(x, y)+1}+\frac{\left|\frac{u}{\mu}\right|^{G(x, y)-1}}{G(x, y)-1}\right] \mathrm{d} x \mathrm{~d} y \leq 1\right\} .
\end{aligned}
$$

From now on we denote the duality pairing between $\mathcal{W}$ and its dual space $\mathcal{W}^{*}$ by $\langle\cdot, \cdot\rangle_{\mathcal{W}}$. Furthermore, we set

$$
G^{+}:=\sup _{(x, y) \in \bar{\Omega}} G(x, y) \quad \text { and } \quad G^{-}:=\inf _{(x, y) \in \bar{\Omega}} G(x, y) .
$$

The following compactness property was proved by Bahrouni et al. [5].

Lemma 3.1. Assume that $G$ is a function of class $C^{1}$ and that $G(x, y) \in(2, N)$ for all $(x, y) \in \bar{\Omega}$. Furthermore, suppose that $s \in\left(1, G^{-}\right)$and $0<\gamma<\frac{N\left(G^{-}-s\right)}{s}$. Then $\mathcal{W}$ is compactly embedded in $L^{s}(\Omega)$.

Now, we define $\rho: \mathcal{W} \rightarrow \mathbb{R}$ by

$$
\begin{aligned}
\rho(u)= & \int_{\Omega} \frac{1}{G(x, y)}\left[\left|\nabla_{x}(u)\right|^{G(x, y)}+|x|^{\gamma}\left|\nabla_{y}(u)\right|^{G(x, y)}\right] \mathrm{d} x \mathrm{~d} y \\
& +\int_{\Omega} A(x, y)\left[\frac{|u|^{G(x, y)+1}}{G(x, y)+1}+\frac{|u|^{G(x, y)-1}}{G(x, y)-1}\right] \mathrm{d} x \mathrm{~d} y .
\end{aligned}
$$

The following lemma will be helpful in later treatments.

Lemma 3.2. Let $u \in \mathcal{W}$, then the following holds:

(i) For $u \neq 0$ we have: $\|u\|_{\mathcal{W}}=a$ if and only if $\rho\left(\frac{u}{a}\right)=1$;

(ii) $\|u\|_{\mathcal{W}}<1$ implies $\|u\|_{\mathcal{W}}^{G^{+}+1} \leq \rho(u) \leq\|u\|_{\mathcal{W}}^{G^{-}-1}$;

(iii) $\|u\|_{\mathcal{W}}>1$ implies $\|u\|_{\mathcal{W}}^{G^{-}-1} \leq \rho(u)$. 
Proof. (i) For every fixed $u \in \mathcal{W}$, the mapping $\lambda \mapsto \rho(\lambda u)$ is a continuous, convex, even function, which is strictly increasing in $[0,+\infty)$. Thus, by the definition of $\rho$ and the equivalent norm given in (3.1), we have

$$
\|u\|_{\mathcal{W}}=a \quad \Longleftrightarrow \quad \rho\left(\frac{u}{a}\right)=1
$$

(ii) Let $u \in \mathcal{W}$ be such that $\|u\|_{\mathcal{W}}<1$, then

$$
\begin{aligned}
& \left\|\nabla_{x} u\right\|_{G(\cdot, \cdot)}<1 \\
& \left\||x|^{\frac{\gamma}{G(x, y)}} \nabla_{y} u\right\|_{G(\cdot, \cdot)}<1, \\
& \left\|u\left(\left|\nabla_{x} G(x, y)\right|+|x|^{\gamma}\left|\nabla_{y} G(x, y)\right|\right)^{\frac{1}{G(x, y)+1}}\right\|_{G(\cdot, \cdot)+1}<1, \\
& \left\|u\left(\left|\nabla_{x} G(x, y)\right|+|x|^{\gamma}\left|\nabla_{y} G(x, y)\right|\right)^{\frac{1}{G(x, y)-1}}\right\|_{G(\cdot, \cdot)-1}<1 .
\end{aligned}
$$

So, by Proposition 2.1, we get the desired result.

(iii) Let $u \in \mathcal{W}$ be such that $\|u\|_{\mathcal{W}}>1$. By (i), we obtain

$$
\begin{aligned}
\rho\left(\frac{u}{\|u\|_{\mathcal{W}}}\right)= & \int_{\Omega} \frac{1}{G(x, y)}\left[\left|\nabla_{x}\left(\frac{u}{\|u\|_{\mathcal{W}}}\right)\right|^{G(x, y)}+|x|^{\gamma}\left|\nabla_{y}\left(\frac{u}{\|u\|_{\mathcal{W}}}\right)\right|^{G(x, y)}\right] \mathrm{d} x \mathrm{~d} y \\
& +\int_{\Omega} A(x, y)\left[\frac{\left|\frac{u}{\|u\|_{\mathcal{W}}}\right|^{G(x, y)+1}}{G(x, y)+1}+\frac{\left|\frac{u}{\|u\|_{\mathcal{W}}}\right|^{G(x, y)-1}}{G(x, y)-1}\right] \mathrm{d} x \mathrm{~d} y=1 .
\end{aligned}
$$

Then, by the mean value theorem, there exist $\left(x_{1}, y_{1}\right),\left(x_{2}, y_{2}\right),\left(x_{3}, y_{3}\right) \in \bar{\Omega}$ depending on $u, G$ and $\Omega$ such that

$$
\begin{aligned}
1= & \frac{1}{\|u\|_{\mathcal{W}}^{G\left(x_{1}, y_{1}\right)}} \int_{\Omega} \frac{1}{G(x, y)}\left[\left|\nabla_{x} u\right|^{G(x, y)}+|x|^{\gamma}\left|\nabla_{y} u\right|^{G(x, y)}\right] \mathrm{d} x \mathrm{~d} y \\
& +\frac{1}{\|u\|_{\mathcal{W}}^{G\left(x_{2}, y_{2}\right)+1}} \int_{\Omega} A(x, y) \frac{|u|^{G(x, y)+1}}{G(x, y)+1} \mathrm{~d} x \mathrm{~d} y \\
& +\frac{1}{\|u\|_{\mathcal{W}}^{G\left(x_{3}, y_{3}\right)-1}} \int_{\Omega} A(x, y) \frac{|u|^{G(x, y)-1}}{G(x, y)-1} \mathrm{~d} x \mathrm{~d} y .
\end{aligned}
$$

Since $\|u\|_{\mathcal{W}}>1$, it follows that

$$
\begin{aligned}
1 \leq & \frac{1}{\|u\|_{\mathcal{W}}^{G_{-}-1}}\left[\int_{\Omega} \frac{1}{G(x, y)}\left[\left|\nabla_{x} u\right|^{G(x, y)}+|x|^{\gamma}\left|\nabla_{y} u\right|^{G(x, y)}\right] \mathrm{d} x \mathrm{~d} y\right] \\
& +\frac{1}{\|u\|_{\mathcal{W}}^{G-1}}\left[\int_{\Omega} A(x, y)\left[\frac{|u|^{G(x, y)+1}}{G(x, y)+1}+\frac{|u|^{G(x, y)-1}}{G(x, y)-1}\right] \mathrm{d} x \mathrm{~d} y\right] .
\end{aligned}
$$

This finishes the proof.

Lemma 3.3. Assume that the assumptions of Lemma 3.1 are fulfilled. Then the following properties hold. 
(i) The functional $\rho$ is of class $C^{1}$, and for all $u, v \in \mathcal{W}$ we have

$$
\begin{aligned}
\left\langle\rho^{\prime}(u), v\right\rangle_{\mathcal{W}}= & \int_{\Omega}\left[\left|\nabla_{x} u\right|^{G(x, y)-2} \nabla_{x} u \nabla_{x} v+|x|^{\gamma}\left|\nabla_{y} u\right|^{G(x, y)-2} \nabla_{y} u \nabla_{y} v\right] \mathrm{d} x \mathrm{~d} y \\
& +\int_{\Omega} A(x, y)|u|^{G(x, y)-3}\left(u^{2}+1\right) u v \mathrm{~d} x \mathrm{~d} y .
\end{aligned}
$$

(ii) The function $\rho^{\prime}: \mathcal{W} \rightarrow \mathcal{W}^{*}$ is coercive, that is, $\frac{\left\langle\rho^{\prime}(u), u\right\rangle_{\mathcal{W}}}{\|u\|_{\mathcal{W}}} \rightarrow+\infty$ as $\|u\|_{\mathcal{W}} \rightarrow+\infty$.

Proof. (i) This follows directly from the definition of $\rho: \mathcal{W} \rightarrow \mathbb{R}$.

(ii) By Lemma 3.2, for $\|u\|_{\mathcal{W}}>1$, we obtain

$$
\left\langle\rho^{\prime}(u), u\right\rangle_{\mathcal{W}} \geq \rho(u) \geq\|u\|_{\mathcal{W}}^{G^{-}-1} .
$$

Then

$$
\frac{\left\langle\rho^{\prime}(u), u\right\rangle_{\mathcal{W}}}{\|u\|_{\mathcal{W}}} \geq\|u\|_{\mathcal{W}}^{G^{-}-2} \rightarrow+\infty
$$

as $\|u\|_{\mathcal{W}} \rightarrow+\infty$ since $G \in(2, N)$ and so $G^{-}>2$.

Lemma 3.4. Let the conditions of Lemma 3.1 be satisfied. Then there exists $\lambda_{1}>0$ such that

$$
\lambda_{1}=\inf _{\substack{u \in \mathcal{W} \\\|u\|_{\mathcal{W}>1}}} \frac{\rho(u)}{\|u\|_{G^{-}-1}^{G^{-}-1}} .
$$

Proof. By Lemma 3.1 there exists $C>0$ such that

$$
\|u\|_{\mathcal{W}} \geq C\|u\|_{G^{--1}} \text { for all } u \in \mathcal{W} .
$$

On the other hand, by Lemma 3.2 , for $\|u\|_{\mathcal{W}}>1$ we have

$$
\rho(u) \geq\|u\|_{\mathcal{W}}^{G^{-}-1}
$$

Combining the above inequalities we obtain

$$
\rho(u) \geq C^{G^{-}-1}\|u\|_{G^{-}-1}^{G^{-}-1} \text { for all } u \in \mathcal{W} \text { with }\|u\|_{\mathcal{W}}>1 .
$$

The proof is now complete.

Lemma 3.5. Assume that the conditions of Lemma 3.1 hold. Then the double phase operator $\rho^{\prime}: \mathcal{W} \rightarrow \mathcal{W}^{*}$ has the following properties:

(i) $\rho^{\prime}$ is a continuous, bounded (that is, it maps bounded sets to bounded sets), and strictly monotone operator.

(ii) $\rho^{\prime}$ is a mapping of type $\left(\mathrm{S}_{+}\right)$, that is, if $u_{n} \rightarrow u$ in $\mathcal{W}$ and $\lim \sup \left\langle\rho^{\prime}\left(u_{n}\right), u_{n}-u\right\rangle_{\mathcal{W}} \leq 0$, then $u_{n} \rightarrow u$ in $\mathcal{W}$.

(iii) $\rho^{\prime}$ is a homeomorphism. 
Proof. (i) From Lemma 3.3 it is clear that $\rho^{\prime}$ is continuous. Next, we are going to prove that $\rho^{\prime}$ maps bounded sets to bounded sets. By Young's inequality, we obtain

$$
\begin{aligned}
& \left\langle\rho^{\prime}(u), v\right\rangle_{\mathcal{W}} \\
& =\int_{\Omega}\left[\left|\nabla_{x} u\right|^{G(x, y)-2} \nabla_{x} u \nabla_{x} v+|x|^{\gamma}\left|\nabla_{y} u\right|^{G(x, y)-2} \nabla_{y} u \nabla_{y} v\right] \mathrm{d} x \mathrm{~d} y \\
& \quad+\int_{\Omega} A(x, y)|u|^{G(x, y)-3}\left(u^{2}+1\right) u v \mathrm{~d} x \mathrm{~d} y \\
& \leq\left(G^{+}-1\right) \int_{\Omega} \frac{1}{G(x, y)}\left[\left|\nabla_{x} u\right|^{G(x, y)}+|x|^{\gamma}\left|\nabla_{y} u\right|^{G(x, y)}\right] \mathrm{d} x \mathrm{~d} y \\
& \quad+\int_{\Omega} \frac{1}{G(x, y)}\left[\left|\nabla_{x} v\right|^{G(x, y)}+|x|^{\gamma}\left|\nabla_{y} v\right|^{G(x, y)}\right] \mathrm{d} x \mathrm{~d} y \\
& \quad+G^{+} \int_{\Omega} A(x, y) \frac{|u|^{G(x, y)+1}}{G(x, y)+1} \mathrm{~d} x \mathrm{~d} y+\int_{\Omega} A(x, y) \frac{|v|^{G(x, y)+1}}{G(x, y)+1} \mathrm{~d} x \mathrm{~d} y \\
& \quad+\left(G^{+}-2\right) \int_{\Omega} A(x, y) \frac{|u|^{G(x, y)-1}}{G(x, y)-1} \mathrm{~d} x \mathrm{~d} y+\int_{\Omega} A(x, y) \frac{|v|^{G(x, y)-1}}{G(x, y)-1} \mathrm{~d} x \mathrm{~d} y \\
& \leq \\
& G^{+} \rho(u)+\rho(v) .
\end{aligned}
$$

Hence, from Lemma 3.2, we get

$$
\left\|\rho^{\prime}(u)\right\|=\sup _{\|v\| \leq 1}\left|\left\langle\rho^{\prime}(u), v\right\rangle_{\mathcal{W}}\right| \leq G^{+} \rho(u)+3,
$$

which implies that $\rho^{\prime}$ maps bounded sets to bounded sets.

The strict monotonicity of $\rho^{\prime}$ is a direct consequence of the well-known Simon inequalities [30, formula (2.2)]

$$
\begin{aligned}
|x-y|^{p} \leq & c_{p}\left(|x|^{p-2} x-|y|^{p-2} y\right) \cdot(x-y) \quad \text { if } p \geq 2, \\
|x-y|^{p} \leq & C_{p}\left[\left(|x|^{p-2} x-|y|^{p-2} y\right) \cdot(x-y)\right]^{\frac{p}{2}} \\
& \times\left(|x|^{p}+|y|^{p}\right)^{\frac{2-p}{p}} \quad \text { if } p \in(1,2)
\end{aligned}
$$

for all $x, y \in \mathbb{R}^{N}$, where $c_{p}$ and $C_{p}$ are positive constants depending only on $p$, see Lindqvist [19, p. 71], Filippucci et al. [14, p. 713], and Pucci et al. [26, p. 14].

(ii) Let $\left\{u_{n}\right\}_{n \geq 1} \subseteq \mathcal{W}$ be a sequence such that

$$
u_{n} \rightarrow u \quad \text { in } \mathcal{W} \quad \text { and } \quad \limsup _{n \rightarrow+\infty}\left\langle\rho^{\prime}\left(u_{n}\right), u_{n}-u\right\rangle_{\mathcal{W}} \leq 0
$$

Then, from (i), we deduce that

$$
\lim _{n \rightarrow+\infty}\left\langle\rho^{\prime}\left(u_{n}\right), u_{n}-u\right\rangle_{\mathcal{W}}=0 .
$$

In view of (3.2) and (3.3), the sequence $\left\{\nabla u_{n}\right\}_{n \geq 1}$ converges in measure to $\nabla u$ in $\Omega$. Then there is a subsequence, still denoted by $\left\{\nabla u_{n}\right\}_{n \geq 1}$, that converges to $\nabla u$ a.e. in $\Omega$. 
First, we have

$$
\begin{aligned}
\left\langle\rho^{\prime}\left(u_{n}\right), u_{n}-u\right\rangle_{\mathcal{W}} & \\
= & \int_{\Omega}\left[\left|\nabla_{x} u_{n}\right|^{G(x, y)-2} \nabla_{x} u_{n} \nabla_{x}\left(u_{n}-u\right)\right. \\
& \left.+|x|^{\gamma}\left|\nabla_{y} u_{n}\right|^{G(x, y)-2} \nabla_{y} u_{n} \nabla_{y}\left(u_{n}-u\right)\right] \mathrm{d} x \mathrm{~d} y \\
& +\int_{\Omega} A(x, y)\left|u_{n}\right|^{G(x, y)-3}\left(u_{n}^{2}+1\right) u_{n}\left(u_{n}-u\right) \mathrm{d} x \mathrm{~d} y \\
= & \int_{\Omega}\left[\left|\nabla_{x} u_{n}\right|^{G(x, y)}+|x|^{\gamma}\left|\nabla_{y} u_{n}\right|^{G(x, y)}\right] \mathrm{d} x \mathrm{~d} y \\
& +\int_{\Omega} A(x, y)\left|u_{n}\right|^{G(x, y)-1}\left(u_{n}^{2}+1\right) \mathrm{d} x \mathrm{~d} y \\
& -\int_{\Omega}\left[\left|\nabla_{x} u_{n}\right|^{G(x, y)-2} \nabla_{x} u_{n} \nabla_{x} u+|x|^{\gamma}\left|\nabla_{y} u_{n}\right|^{G(x, y)-2} \nabla_{y} u_{n} \nabla_{y} u\right] \mathrm{d} x \mathrm{~d} y \\
& -\int_{\Omega} A(x, y)\left|u_{n}\right|^{G(x, y)-3}\left(u_{n}^{2}+1\right) u_{n} u \mathrm{~d} x \mathrm{~d} y .
\end{aligned}
$$

Then, by applying Young's inequality to the right-hand side of the last equation, we obtain

$$
\begin{aligned}
\left\langle\rho^{\prime}\left(u_{n}\right), u_{n}-u\right\rangle_{\mathcal{W}} & \int_{\Omega}\left[\left|\nabla_{x} u_{n}\right|^{G(x, y)}+|x|^{\gamma}\left|\nabla_{y} u_{n}\right|^{G(x, y)}\right] \mathrm{d} x \mathrm{~d} y \\
& +\int_{\Omega} A(x, y)\left|u_{n}\right|^{G(x, y)-1}\left(u_{n}^{2}+1\right) \mathrm{d} x \mathrm{~d} y \\
& -\int_{\Omega} \frac{G(x, y)-1}{G(x, y)}\left[\left|\nabla_{x} u_{n}\right|^{G(x, y)}+|x|^{\gamma}\left|\nabla_{y} u_{n}\right|^{G(x, y)}\right] \mathrm{d} x \mathrm{~d} y \\
& -\int_{\Omega} \frac{1}{G(x, y)}\left[\left|\nabla_{x} u\right|^{G(x, y)}+|x|^{\gamma}\left|\nabla_{y} u\right|^{G(x, y)}\right] \mathrm{d} x \mathrm{~d} y \\
& -\int_{\Omega} A(x, y) \frac{G(x, y)}{G(x, y)+1}\left|u_{n}\right|^{G(x, y)+1} \mathrm{~d} x \mathrm{~d} y \\
& -\int_{\Omega} A(x, y) \frac{1}{G(x, y)+1}|u|^{G(x, y)+1} \mathrm{~d} x \mathrm{~d} y \\
& -\int_{\Omega} A(x, y) \frac{G(x, y)-2}{G(x, y)-1}\left|u_{n}\right|^{G(x, y)-1} \mathrm{~d} x \mathrm{~d} y \\
& -\int_{\Omega} A(x, y) \frac{1}{G(x, y)-1}|u|^{G(x, y)-1} \mathrm{~d} x \mathrm{~d} y \\
\geq & \rho\left(u_{n}\right)-\rho(u) .
\end{aligned}
$$


This finally gives

$$
\left\langle\rho^{\prime}\left(u_{n}\right), u_{n}-u\right\rangle_{\mathcal{W}} \geq \rho\left(u_{n}\right)-\rho(u) .
$$

Combining (3.4) and (3.5) leads to

$$
\lim _{n \rightarrow+\infty} \rho\left(u_{n}\right) \leq \rho(u) .
$$

On the other hand, it follows from Fatou's lemma that

$$
\liminf _{n \rightarrow+\infty} \rho\left(u_{n}\right) \geq \rho(u) .
$$

Thus, we have that

$$
\lim _{n \rightarrow+\infty} \rho\left(u_{n}\right)=\rho(u),
$$

which implies that the family of continuous functions

$$
\begin{aligned}
& \left\{\frac{1}{G(x, y)}\left[\left|\nabla_{x} u_{n}\right|^{G(x, y)}+|x|^{\gamma}\left|\nabla_{y} u_{n}\right|^{G(x, y)}\right]\right. \\
& \left.\quad+A(x, y)\left[\frac{\left|u_{n}\right|^{G(x, y)+1}}{G(x, y)+1}+\frac{\left|u_{n}\right|^{G(x, y)-1}}{G(x, y)-1}\right]\right\}_{n \geq 1}
\end{aligned}
$$

turns out to be equicontinuous on $\Omega$. Since

$$
\begin{aligned}
& \frac{1}{G(x, y)}\left|\nabla_{x}\left(u_{n}-u\right)\right|^{G(x, y)}+\frac{1}{G(x, y)}|x|^{\gamma}\left|\nabla_{y}\left(u_{n}-u\right)\right|^{G(x, y)} \\
& \quad+A(x, y)\left[\frac{\left|u_{n}-u\right|^{G(x, y)+1}}{G(x, y)+1}+\frac{\left|u_{n}-u\right|^{G(x, y)-1}}{G(x, y)-1}\right] \\
& \quad \leq \frac{C}{G(x, y)}\left(\left|\nabla_{x} u_{n}\right|^{G(x, y)}+\left|\nabla_{x} u\right|^{G(x, y)}\right) \\
& \quad+\frac{C}{G(x, y)}\left(\left|\nabla_{y} u_{n}\right|^{G(x, y)}+\left|\nabla_{y} u\right|^{G(x, y)}\right) \\
& \quad+C A(x, y)\left(\frac{\left|u_{n}\right|^{G(x, y)+1}}{G(x, y)+1}+\frac{|u|^{G(x, y)+1}}{G(x, y)+1}\right) \\
& \quad+C A(x, y)\left(\frac{\left|u_{n}\right|^{G(x, y)-1}}{G(x, y)-1}+\frac{|u|^{G(x, y)-1}}{G(x, y)-1}\right)
\end{aligned}
$$

with positive $C$, the integrals of the family

$$
\begin{aligned}
& \left\{\frac{1}{G(x, y)}\left|\nabla_{x}\left(u_{n}-u\right)\right|^{G(x, y)}+\frac{1}{G(x, y)}|x|^{\gamma}\left|\nabla_{y}\left(u_{n}-u\right)\right|^{G(x, y)}\right. \\
& \left.\quad+A(x, y)\left[\frac{\left|u_{n}-u\right|^{G(x, y)+1}}{G(x, y)+1}+\frac{\left|u_{n}-u\right|^{G(x, y)-1}}{G(x, y)-1}\right]\right\}_{n \geq 1}
\end{aligned}
$$

are also equicontinuous on $\Omega$ and therefore

$$
\lim _{n \rightarrow+\infty} \rho\left(u_{n}-u\right)=0 .
$$

It follows, by Proposition 2.2, that

$$
u_{n} \rightarrow u \quad \text { in } \mathcal{W} \text {. }
$$


(iii) By strict monotonicity, $\rho^{\prime}$ is an injection. On the other hand, using Lemma 3.3 and the MintyBrowder theorem, $\rho^{\prime}$ is a surjection. Hence $\rho^{\prime}$ has an inverse mapping $\left(\rho^{\prime}\right)^{-1}: \mathcal{W}^{*} \rightarrow \mathcal{W}$. Therefore, in order to complete the proof of (iii), it suffices to prove that $\left(\rho^{\prime}\right)^{-1}$ is continuous. If $f_{n}, f \in$ $\mathcal{W}^{*}, f_{n} \rightarrow f$, letting $u_{n}=\left(\rho^{\prime}\right)^{-1}\left(f_{n}\right), u=\left(\rho^{\prime}\right)^{-1}(f)$, then $\rho^{\prime}\left(u_{n}\right)=f_{n}, \rho^{\prime}(u)=f$. Note that $\left\{u_{n}\right\}_{n \geq 1}$ is bounded in $\mathcal{W}$. Without loss of generality, we can assume that $u_{n} \rightarrow u_{0}$ in $\mathcal{W}$. We conclude from $f_{n} \rightarrow f$ that

$$
\lim _{n \rightarrow+\infty}\left\langle\rho^{\prime}\left(u_{n}\right)-\rho^{\prime}\left(u_{0}\right), u_{n}-u_{0}\right\rangle_{\mathcal{W}}=\lim _{n \rightarrow+\infty}\left\langle f_{n}-f, u_{n}-u_{0}\right\rangle_{\mathcal{W}}=0 .
$$

Since $\rho^{\prime}$ is of type $\left(\mathrm{S}_{+}\right)$, we know that $u_{n} \rightarrow u_{0}$ in $\mathcal{W}$ and so $u_{n} \rightarrow u$ in $\mathcal{W}$.

\section{Existence of a solution}

We suppose the following hypotheses on the reaction term in (1.1).

(H) $f: \Omega \times \mathbb{R} \times \mathbb{R}^{N} \rightarrow \mathbb{R}$ is a Carathéodory function with $f((x, y), 0,0) \neq 0$ for a. a. $(x, y) \in \Omega$ such that the following holds:

(i) there exists $a \in L^{\infty}\left(\Omega \times \mathbb{R}^{N}\right)$ such that

$$
|f((x, y), s, \xi)| \leq a((x, y), \xi)\left(1+|s|^{G^{-}-1}\right)
$$

for a. a. all $(x, y) \in \Omega$, for all $s \in \mathbb{R}$ and for all $\xi \in \mathbb{R}^{N}$;

(ii) there exists $\vartheta \in\left(0, \lambda_{1}\right)$ such that

$$
\limsup _{s \rightarrow+\infty} \frac{f((x, y), s, \xi)}{s^{G^{-}-1}} \leq \vartheta \quad \text { uniformly for a. a. }(x, y) \in \Omega
$$

and for all $\xi \in \mathbb{R}^{N}$ with $\lambda_{1}$ given in Lemma 3.4.

We say that $u \in \mathcal{W}$ is a weak solution of problem (1.1) if

$$
\begin{aligned}
\int_{\Omega} & {\left[\left|\nabla_{x} u\right|^{G(x, y)-2} \nabla_{x} u \nabla_{x} \varphi+|x|^{\gamma}\left|\nabla_{y} u\right|^{G(x, y)-2} \nabla_{y} u \nabla_{y} \varphi\right] \mathrm{d} x \mathrm{~d} y } \\
& +\int_{\Omega} A(x, y)|u|^{G(x, y)-3}\left(u^{2}+1\right) u \varphi \mathrm{d} x \mathrm{~d} y \\
= & \int_{\Omega} f((x, y), u, \nabla u) v \mathrm{~d} x \mathrm{~d} y .
\end{aligned}
$$

is satisfied for all $\varphi \in \mathcal{W} \backslash\{0\}$.

Now we are in the position to state our main existence result.

Theorem 4.1. Suppose that conditions $(\mathrm{H})(\mathrm{i})$, (ii) are fulfilled. Moreover, assume that $G$ is a function of class $C^{1}$ and that $G(x, y) \in(2, N)$ for all $(x, y) \in \Omega$. Furthermore, suppose that $s \in\left(1, G^{-}\right)$and $0<\gamma<\frac{N\left(G^{-}-s\right)}{s}$. Then problem (1.1) admits at least one nontrivial weak solution.

Proof. Let $N_{f}: \mathcal{W} \subseteq L^{G^{-}-1} \rightarrow L^{\left(G^{-}-1\right)^{\prime}} \subseteq \mathcal{W}^{*}$ be the Nemytskij operator corresponding to the nonlinearity $f: \Omega \times \mathbb{R} \times \mathbb{R}^{N} \rightarrow \mathbb{R}$ which is compact by Lemma 3.1. Now we define the operator $I: \mathcal{W} \rightarrow \mathcal{W}^{*}$ by

$$
I(u)=\rho^{\prime}(u)-N_{f}(u) .
$$


Because of the growth condition $\mathrm{H}(\mathrm{i})$ and Lemma 3.5(i) we know that $I: \mathcal{W} \rightarrow \mathcal{W}^{*}$ maps bounded sets into bounded sets. Let us now prove that $I$ is pseudomonotone in the sense of Definition 2.3. To this end, let $\left\{u_{n}\right\}_{n \geq 1} \subseteq \mathcal{W}$ be a sequence such that

$$
u_{n} \rightarrow u \quad \text { in } \mathcal{W} \quad \text { and } \quad \limsup _{n \rightarrow+\infty}\left\langle I\left(u_{n}\right), u_{n}-u\right\rangle_{\mathcal{W}} \leq 0
$$

Recall that

$$
\left\langle I\left(u_{n}\right), u_{n}-u\right\rangle=\left\langle\rho^{\prime}\left(u_{n}\right), u_{n}-u\right\rangle-\int_{\Omega} f\left((x, y), u_{n}, \nabla u_{n}\right)\left(u_{n}-u\right) \mathrm{d} x \mathrm{~d} y .
$$

By Lemma 3.1 we know that

$$
u_{n} \rightarrow u \text { in } L^{G^{-}-1}(\Omega)
$$

since $G^{-}-1<G^{-}$. Moreover, hypothesis $\mathrm{H}(\mathrm{i})$ implies that

$$
\left\{N_{f}\left(u_{n}\right)\right\}_{n \geq 1} \subseteq L^{\left(G^{-}-1\right)^{\prime}}(\Omega) \text { is bounded. }
$$

From these facts it is clear that

$$
\int_{\Omega} f\left((x, y), u_{n}, \nabla u_{n}\right)\left(u_{n}-u\right) \mathrm{d} x \mathrm{~d} y \rightarrow 0 \quad \text { as } n \rightarrow+\infty .
$$

Therefore, passing to the limit in (4.2) and using (4.1) as well as (4.3) leads to

$$
\limsup _{n \rightarrow+\infty}\left\langle\rho^{\prime}\left(u_{n}\right), u_{n}-u\right\rangle=\limsup _{n \rightarrow+\infty}\left\langle I\left(u_{n}\right), u_{n}-u\right\rangle \leq 0 .
$$

From Lemma 3.5 we know that $\rho^{\prime}$ fulfills the $\left(\mathrm{S}_{+}\right)$-property and so we conclude, in view of $(4.1)$ and (4.4), that

$$
u_{n} \rightarrow u \quad \text { in } \mathcal{W} .
$$

Thus, because of the continuity of $I: \mathcal{W} \rightarrow \mathcal{W}^{*}$, we have $I\left(u_{n}\right) \rightarrow I(u)$ in $\mathcal{W}^{*}$ which proves that $I$ is pseudomonotone.

Next, we have to show that the operator $I: \mathcal{W} \rightarrow \mathcal{W}^{*}$ is coercive, that is,

$$
\lim _{\|u\|_{\mathcal{W}} \rightarrow \infty} \frac{\langle I(u), u\rangle_{\mathcal{W}}}{\|u\|_{\mathcal{W}}}=\infty
$$

Note that hypothesis (H)(ii) implies that for a given $\varepsilon>0$ there exists $M=M(\varepsilon)>1$ such that

$$
f(x, s, \xi) s \leq(\vartheta+\varepsilon) s^{G^{-}-1}
$$

for a. a. $x \in \Omega$, for all $s \geq M$ and for all $\xi \in \mathbb{R}^{N}$.

Let $u \in \mathcal{W}$ be such that $\|u\|>M>1$. Applying Lemma 3.3, (4.5), Lemmas 3.4 and 3.2(iii) we get

$$
\begin{aligned}
\langle I(u), u\rangle & =\left\langle\rho^{\prime}(u), u\right\rangle-\int_{\Omega} f((x, y), u, \nabla u) u \mathrm{~d} x \mathrm{~d} y \\
& \geq \rho(u)-(\vartheta+\epsilon) \int_{\Omega}|u|^{G^{-}-1} \mathrm{~d} x \mathrm{~d} y \\
& =\rho(u)-(\vartheta+\epsilon)\|u\|_{G^{-}-1}^{G^{-}-1} \\
& \geq\left(1-\frac{\vartheta+\epsilon}{\lambda_{1}}\right) \rho(u) \\
& \geq\left(1-\frac{\vartheta+\epsilon}{\lambda_{1}}\right)\|u\|_{\mathcal{W}}^{G^{-}-1} .
\end{aligned}
$$

Choosing $\varepsilon \in\left(0, \lambda_{1}-\vartheta\right)$ proves that $I: \mathcal{W} \rightarrow \mathcal{W}^{*}$ is coercive. 
To sum up, we have shown that the operator $I: \mathcal{W} \rightarrow \mathcal{W}^{*}$ is bounded, pseudomonotone and coercive. Therefore, the main theorem on pseudomonotone operators, see Theorem 2.4 , provides $u \in \mathcal{W}, u \neq 0$ (since $f(x, 0,0) \neq 0$ ), such that $I(u)=0$. By the definition of $I$, the function $u$ turns out to be a nontrivial weak solution of problem (1.1) which completes the proof.

\section{Concluding remarks, perspectives, and open problems}

(i) The mathematical analysis carried out in this paper considers the unbalanced energy

$$
\mathcal{W} \ni u \mapsto \int_{\Omega} \frac{1}{G(x, y)}\left[\left|\nabla_{x}(u)\right|^{G(x, y)}+|x|^{\gamma}\left|\nabla_{y}(u)\right|^{G(x, y)}\right] \mathrm{d} x \mathrm{~d} y
$$

with the associated differential operator

$$
\Delta_{G(x, y)} u=\sum_{i=1}^{n}\left(\left|\nabla_{x}\right|^{G(x, y)-2} u_{x_{i}}\right)_{x_{i}}+|x|^{\gamma} \sum_{i=1}^{m}\left(\left|\nabla_{y}\right|^{G(x, y)-2} u_{y_{i}}\right)_{y_{i}} .
$$

It appears to be worth to further investigate patterns described by the variational integral

$$
\int_{\Omega}\left(\left|\nabla_{x} u\right|^{G(x, y)}+|x|^{\gamma}\left|\nabla_{y} u\right|^{G(x, y)}\right) \mathrm{d} z
$$

with corresponding anisotropic Baouendi-Grushin operator

$$
\operatorname{div}_{x}\left(G(x, y)\left|\nabla_{x}\right|^{G(x, y)-2} \nabla_{x}\right)+\operatorname{div}_{y}\left(G(x, y)|x|^{\gamma}\left|\nabla_{y}\right|^{G(x, y)-2} \nabla_{y}\right) .
$$

(ii) We remark that since both $\rho$ and the energy functional defined in (4.6) have a degenerate action on the set where the gradient vanishes, it is a natural question to study what happens if the integrand is modified in such a way that, if $|\nabla u|$ is also small, there exists an imbalance between the two terms of every integrand.

(iii) The compactness property established in Lemma 3.1 plays a key role in the proof of several crucial properties such as: coercivity of $\rho^{\prime}$ (Lemma 3.3), existence of a principal eigenvalue associated with the Rayleigh quotient (Lemma 3.4), as well as in the proof of the main result established in Theorem 4.1. This compactness property is established in a subcritical setting, which corresponds to the hypothesis $s<G^{-}$, where $s$ describes the growth of the right-hand side of problem (1.1). In fact, Theorem 4.1 remains true if $s$ is replaced with a variable coefficient $s(x)$, provided that $s^{+}<G^{-}$. We do not have any knowledge about the behaviour in the almost critical case that arises in the following situation: there exists $x_{0} \in \Omega$ such that $s\left(x_{0}\right)=G^{-}$and $s(x)<G^{-}$for all $x \in \Omega \backslash\left\{x_{0}\right\}$.

(iv) It is worth noting that the study of nonlinear boundary value problems involving the magnetic Baouendi-Grushin operator [3] are of real interest for mathematical physics patterns. This operator is

$$
G_{\mathcal{A}}:=-\left(\nabla_{G}+i \beta \mathcal{A}_{0}\right)^{2} \quad \text { for }-\frac{1}{2} \leq \beta \leq \frac{1}{2}
$$

where

$$
\begin{aligned}
\mathcal{A}_{0} & =\left(\mathcal{A}_{1}, \mathcal{A}_{2}, \mathcal{A}_{3}, \mathcal{A}_{4}\right)=\left(-\frac{\partial_{y} d}{d}, \frac{\partial_{x} d}{d},-2 y \frac{\partial_{t} d}{d}, 2 x \frac{\partial_{t} d}{d}\right), \\
\nabla_{G} & =\left(\partial_{x}, \partial_{y}, 2 x \partial_{t}, 2 y \partial_{t}\right),
\end{aligned}
$$

with $z=(x, y),|z|=\sqrt{x^{2}+y^{2}}$, and $d(z, t)=\left(|z|^{4}+t^{2}\right)^{1 / 4}$ is the Kaplan distance. 
Open Access. This article is licensed under a Creative Commons Attribution 4.0 International License, which permits use, sharing, adaptation, distribution and reproduction in any medium or format, as long as you give appropriate credit to the original author(s) and the source, provide a link to the Creative Commons licence, and indicate if changes were made. The images or other third party material in this article are included in the article's Creative Commons licence, unless indicated otherwise in a credit line to the material. If material is not included in the article's Creative Commons licence and your intended use is not permitted by statutory regulation or exceeds the permitted use, you will need to obtain permission directly from the copyright holder. To view a copy of this licence, visit http://creativecommons.org/licenses/by/4.0/.

Publisher's Note Springer Nature remains neutral with regard to jurisdictional claims in published maps and institutional affiliations.

\section{References}

[1] Abdellaoui, B., Peral, I.: On quasilinear elliptic equations related to some Caffarelli-Kohn-Nirenberg inequalities. Commun. Pure Appl. Anal. 2(4), 539-566 (2003)

[2] Adimurthi, A., Chaudhuri, N., Ramaswamy, M.: An improved Hardy-Sobolev inequality and its application. Proc. Am. Math. Soc. 130(2), 489-505 (2002)

[3] Aermark, L., Laptev, A.: Hardy's inequality for the Grushin operator with a magnetic field of Aharanov-Bohm type. Algebra Anal. 23(2), (2011), 1-8 (Russian); St. Petersb. Math. J. 23 (2012), no. 2, 203-208 (English translation)

[4] Bahrouni, A., Rădulescu, V.D., Repovš, D.D.: A weighted anisotropic variant of the Caffarelli-Kohn-Nirenberg inequality and applications. Nonlinearity 31(4), 1516-1534 (2018)

[5] Bahrouni, A., Rădulescu, V.D., Repovš, D.D.: Double phase transonic flow problems with variable growth: nonlinear patterns and stationary waves. Nonlinearity 32(7), 2481-2495 (2019)

[6] Bahrouni, A., Repovš, D.D.: Existence and nonexistence of solutions for $p(x)$-curl systems arising in electromagnetism. Complex Var. Elliptic Equ. 63(2), 292-301 (2018)

[7] Baouendi, M.S.: Sur une classe d'opérateurs elliptiques dégénérés. Bull. Soc. Math. France 95, 45-87 (1967)

[8] Baroni, P., Colombo, M., Mingione, G.: Nonautonomous functionals, borderline cases and related function classes. Algebra Anal. 27(3), 6-50 (2015)

[9] Beck, L., Mingione, G.: Lipschitz bounds and non-uniform ellipticity. Commun. Pure Appl. Math. 73(5), 944-1034 (2020)

[10] Caffarelli, L., Kohn, R., Nirenberg, L.: First order interpolation inequalities with weights. Compos. Math. 53(3), 259-275 (1984)

[11] Catrina, F., Wang, Z.-Q.: On the Caffarelli-Kohn-Nirenberg inequalities: sharp constants, existence (and nonexistence), and symmetry of extremal functions. Commun. Pure Appl. Math. 54(2), 229-258 (2001)

[12] Colasuonno, F., Pucci, P.: Multiplicity of solutions for $p(x)$-polyharmonic elliptic Kirchhoff equations. Nonlinear Anal. 74(17), 5962-5974 (2011)

[13] Colombo, M., Mingione, G.: Bounded minimisers of double phase variational integrals. Arch. Ration. Mech. Anal. 218(1), 219-273 (2015)

[14] Filippucci, R., Pucci, P., Rădulescu, V.D.: Existence and non-existence results for quasilinear elliptic exterior problems with nonlinear boundary conditions. Commun. Part. Differ. Equ. 33, 706-717 (2008)

[15] Franchi, B., Tesi, M.C.: A finite element approximation for a class of degenerate elliptic equations. Math. Comput. 69, 41-63 (1999)

[16] Gasiński, L., Papageorgiou, N.S.: Nonlinear Analysis. Chapman \& Hall/CRC, Boca Raton (2006)

[17] Grushin, V.V.: On a class of hypoelliptic operators. Math. USSR-SB 12, 458-476 (1970)

[18] Hájek, P., Santalucía, V.M., Vanderwerff, J., Zizler, V.: Biorthogonal Systems in Banach Spaces. Springer, New York (2008)

[19] Lindqvist, P.: Notes on the p-Laplace Equation, Report. University of Jyväskylä Department of Mathematics and Statistics, vol. 102. University of Jyväskylä, Jyväskylä (2006)

[20] Morawetz, C.: On the non-existence of continuous transonic flows past profiles. I. Commun. Pure Appl. Math. 9, 45-68 (1956)

[21] Morawetz, C.: On the non-existence of continuous transonic flows past profiles. II. Commun. Pure Appl. Math. 10, 107-131 (1957)

[22] Morawetz, C.: On the non-existence of continuous transonic flows past profiles. III. Commun. Pure Appl. Math. 11, 129-144 (1958)

[23] Musielak, J.: Orlicz Spaces and Modular Spaces. Springer, Berlin (1983)

[24] Papageorgiou, N.S., Rădulescu, V.D., Repovš, D.D.: Double-phase problems with reaction of arbitrary growth. Z. Angew. Math. Phys. 69(4), 108 (2018) 
[25] Papageorgiou, N.S., Rădulescu, V.D., Repovš, D.D.: Double-phase problems and a discontinuity property of the spectrum. Proc. Am. Math. Soc. 147, 2899-2910 (2019)

[26] Pucci, P., Xiang, M., Zhang, B.: Multiple solutions for nonhomogeneous Schrödinger-Kirchhoff type equations involving the fractional $p$-Laplacian in $\mathbb{R}^{N}$. Calc. Var. Part. Differ. Equ. 54(3), 2785-2806 (2015)

[27] Rădulescu, V.D.: Nonlinear elliptic equations with variable exponent: old and new. Nonlinear Anal. 121, 336-369 (2015)

[28] Rădulescu, V.D.: Isotropic and anisotropic double-phase problems: old and new. Opuscu. Math. 39, 259-279 (2019)

[29] Rădulescu, V.D., Repovš, D.D.: Partial Differential Equations with Variable Exponents. CRC Press, Boca Raton (2015)

[30] Simon, J., Régularité de la solution d'une équation non linéaire dans $\mathbb{R}^{N}$. In: Journées d'Analyse Non Linéaire (Proc. Conf. Besançon, 1977), vol. 665, pp. 205-227. Springer, Berlin (1978)

[31] Zhang, Q., Rădulescu, V.D.: Double phase anisotropic variational problems and combined effects of reaction and absorption terms. J. Math. Pures Appl. 118(9), 159-203 (2018)

Anouar Bahrouni

Mathematics Department, Faculty of Sciences

University of Monastir

5019 Monastir

Tunisia

e-mail: bahrounianouar@yahoo.fr

Vicenţiu D. Rădulescu

Faculty of Applied Mathematics

AGH University of Science and Technology

30-059 Kraków

Poland

Vicenţiu D. Rădulescu

Department of Mathematics

University of Craiova

200585 Craiova

Romania

e-mail: radulescu@inf.ucv.ro

Patrick Winkert

Technische Universität Berlin, Institut für Mathematik

Straße des 17 Juni 136

10623 Berlin

Germany

e-mail: winkert@math.tu-berlin.de

(Received: January 6, 2020; accepted: September 28, 2020) 